\title{
An overlooked cause of posterior knee pain: ultrasound imaging and guided injection for proximal lateral gastrocnemius tendon sprain
}

\author{
Ke-Vin Chang'1, Wei-Ting Wu ${ }^{1}$, Levent Özçakar ${ }^{2}$ \\ ${ }^{1}$ Department of Physical Medicine and Rehabilitation, National Taiwan University Hospital, Bei-Hu Branch and Na- \\ tional Taiwan University College of Medicine, Taipei, Taiwan, ${ }^{2}$ Department of Physical and Rehabilitation Medicine, \\ Hacettepe University Medical School, Ankara, Turkey
}

\section{To the editor}

A 66-year-old female had acute onset of right posterior knee pain after hiking, aggravated while she attempted to squat. She stated that the pain ( 7 over 10 on visual analogue scale) had originated from the posterior lateral corner of the popliteal fossa. The physical examination revealed vague tenderness on the distal posterior femoral condyle. Ultrasound examination showed normal findings over the lateral collateral ligament, biceps femoris tendon, popliteal tendon and posterior cruciate ligament. The transducer was later relocated on her painful point almost two finger-width cranial to the knee joint line. Using the heel-toe maneuver, the transducer was able to insonate the posterior femoral condyle in the longitudinal axis, where the proximal tendon of the lateral gastrocnemius muscle attached. Compared with the asymptomatic knee, the painful tendon appeared to have lost its fibrillary pattern and become thickened and hypoechoic. Part of the swollen tendon protruded into the overlying fat pad whereby intra-tendinous vascularity was also visualized (fig 1). Under the impression of proximal gastrocnemius tendon sprain, ultrasound guided injection was performed using $4 \mathrm{~mL} 25 \%$ dextrose to target the tendon's short axis through the in-plane approach (Video 1, on the journal site). The patient was free of pain at follow-up two weeks after the intervention.

Proximal lateral gastrocnemius tendon injury is an overlooked cause of posterior knee pain. Its clinical pres-

Received 07.05.2020 Accepted 30.06.2020

Med Ultrason

2020, Vol. 22, No 3, 377-378, DOI: 10.11152/mu-2610,

Corresponding author: Ke-Vin Chang, MD, PhD

Department of Physical Medicine and

Rehabilitation, National Taiwan University

Hospital, Bei-Hu Branch and National Taiwan

University College of Medicine, Taipei, Taiwan

E-mail:kvchang011@gmail.com; pattap@pchome.com.tw

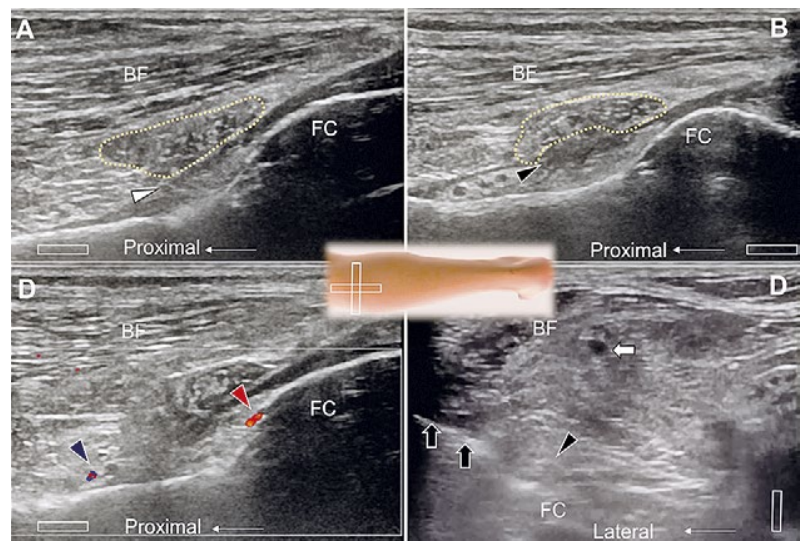

Fig 1. The proximal lateral gastrocnemius tendon (white/black arrowheads) at the asymptomatic (A) and painful (B) sides. Power Doppler imaging (C) and guided injection (D) of the sprained tendon. Blue arrowhead: superior lateral genicular artery; red arrowhead: intra-tendinous vascularity; white arrow: common peroneal nerve; black arrows: needle; dashed area: fat pad. BF: biceps femoris muscle; FC: femoral condyle.

entation is hardly differentiated from lateral collateral ligament or biceps femoris tendon sprain. However, as the proximal gastrocnemius tendon attaches on the distal femur; its pain is more deeply located and can also be aggravated by flexion of the knee.

Ultrasound is helpful in the diagnosis of proximal lateral gastrocnemius tendon injury $[1,2]$. Due to the angulation of the posterior femoral condyle, the tendon is vulnerable to anisotropy during ultrasound examination. As such, the examiner needs to compress the cranial edge of the transducer to position it more parallel to the tendon's long axis. Between the tendon and the overlying biceps femoris muscle there is a fat pad which may sometimes be misrecognized as a diseased tendon. Gentle flexion of the knee can assist in differentiating the border of the fat pad. Of note, the superior lateral genicular artery usually courses through the cranial end of the tendon and should not be interpreted as an increase in intra-tendinous vascularity. 
Ultrasound guided dextrose injections have been proven effective in tendinopathies $[3,4]$ and are suitable for treating proximal gastrocnemius tendon sprain as well. When performing the injection, we suggest to target the short-axis of the tendon for identifying the common peroneal nerve [5], preventing unnecessary nerve damage.

Funding information: The current research project was supported by (1) National Taiwan University Hospital, Bei-Hu Branch; (2) Ministry of Science and Technology (MOST 106-2314-B-002-180-MY3 and 109-2314B-002 -114 -MY3); and (3) Taiwan Society of Ultrasound in Medicine.

\section{References}

1. Chang KV, Wu WT, Özçakar L. Ultrasound-guided diagnosis and intervention for painful knees: so- noanatomy revisited. Pain Manag 2018;8:255258.

2. Özçakar L, Kara M, Chang KV, et al. EURO-MUSCULUS/ USPRM. Basic scanning protocols for knee. Eur J Phys Rehabil Med 2015;51:641-646.

3. Chang KV, Wu WT, Özçakar L. An Underdiagnosed Cause of Anterior Lateral Hip Pain: Ultrasound Imaging and Guided Injection for Rectus Femoris Indirect Tendon Strain. Pain Med 2018;19:1878-1879.

4. Hung CY, Chang KV, Özçakar L. Snapping Hip due to Gluteus Medius Tendinopathy: Ultrasound Imaging in the Diagnosis and Guidance for Prolotherapy. Pain Med 2015;16:2040-2041.

5. Hung CY, Hsiao MY, Özçakar L, et al. Sonographic Tracking of the Lower Limb Peripheral Nerves: A Pictorial Essay and Video Demonstration. Am J Phys Med Rehabil 2016;95:698-708.

\section{Ultrasound Lumbar Spine Clinical Training Phantom: Which is the Best Embedding Medium?}

\section{Zainab M.R. Al Lawati, Ourania Varsou}

Anatomy Facility, School of Life Sciences, University of Glasgow, Scotland UK

\section{To the Editor,}

Lumbar puncture and central neuraxial blocks (e.g. epidural) are common clinical procedures. These were traditionally performed using anatomical landmarks and haptic feedback [1]. However, landmarks are not always palpable (e.g. in patients with high BMI or edema) [1,2]. Ultrasound is increasingly used to guide healthcare professionals during such procedures. Ultrasound improves success rate on first attempt for central neuraxial blocks [1] and aids identification of lumbar puncture landmarks in patients with high BMI [2]. Additionally, ultrasound is characterized by real-time non-invasive scanning without any ionizing radiation $[1,3]$.

Ultrasound phantoms (i.e. synthetic devices simulating human structures) are used for demonstration/clinical training [4]. Phantoms are not associated with inci-

Received 19.06.2020 Accepted 16.07.2020

Med Ultrason

2020, Vol. 22, No 3, 378-379, DOI: 10.11152/mu-2684,

Corresponding author: Ourania Varsou, MD

School of Life Sciences, Anatomy Facility

Thomson Building, University Avenue,

Glasgow G12 8QQ, Scotland UK

Phone +44 (0)141330 7726

E-mail: o.varsou@googlemail.com dental findings [3] and are easily accessible with good anatomical fidelity [4]. Their custom-made nature allows for tailored scanning and repetitive interventional training $[3,4]$. Novices can acquire clinical skills when using phantoms for training [5]. However, commercial phantoms can be costly to purchase $[3,4]$.

We developed three ultrasound lumbar spine clinical training phantoms using different embedding media simulating adipose tissue; i) agar agar (Special Ingredients; UK) prepared as per the manufacturer's recommendations and using an adapted protocol from Scheppler et al [6] ; ii) agar agar with psyllium husk (Planète au Naturel; France) prepared as per the manufacturer's recommendations using a layered approach for embedding; and iii) artificial gelatin number 0 (Humimic Medical; USA) prepared as per the manufacturer's recommendations. Standard latex tubing was used to simulate the dura mater within a pre-made model of the lumbar spine (Zgood Dental 1:1.5 Medical Spine Lumbar Disc Herniation Model; Zgood; China).

The agar agar phantom was the most affordable, whereas the agar agar with psyllium husk had the best background echogenicity. The artificial gelatin phantom had the longest shelf life allowing for repetitive use. Overall, each phantom had advantages and disadvan- 
Table I. Characteristics of the three different lumbar spine phantoms

\begin{tabular}{llll}
\hline Characteristics & Agar Agar & Agar Agar with Psyllium Husk & Artificial Gelatin \\
\hline Development & Easy & Moderate & Moderate \\
Development duration & $2 \mathrm{hrs} 45 \mathrm{~min}$ & $3 \mathrm{hrs} 15 \mathrm{~min}$ & $3 \mathrm{hrs} 30 \mathrm{~min}$ \\
Anatomical fidelity & Moderate & Good & Moderate \\
Tissue firmness & Firm & Average & Firm \\
Background echogenicity & Moderate & Good & Poor \\
Repetitive use & Once & Once & Several times \\
Shelf life & $\sim 45 \mathrm{~min}$ & $\sim 45 \mathrm{~min}$ & Long lasting \\
Cost* & $£ 30.93$ & $£ 40.92$ & $£ 41.82$ \\
Use alongside cadaveric tissue & Yes & Yes & Yes \\
\hline
\end{tabular}

* Indicates cost in pound sterling, including VAT, at the time of the experiment (September 2019) calculated based on the purchase price of each item and not including any lab equipment.

tages (Table I) with the final decision as to which one to adopt guided by its ultimate purpose. In the future, we will replace the latex tubing with an alternative that does not pose a potential allergy risk and we will start simulating additional anatomical structures (e.g. ligaments). We will also invite healthcare professionals to try the phantoms and provide feedback.

\section{References}

1. Karmakar MK. Ultrasound for central neuraxial blocks. Tech Reg Anesth Pain Manag 2009;13:161-170.

2. Stiffler KA, Jwayyed S, Wilber ST, Robinson A. The use of ultrasound to identify pertinent landmarks for lumbar puncture. Am J Emerg Med 2007;25:331-334.
3. Varsou O. The use of ultrasound in educational settings: what should we consider when implementing this technique for visualisation of anatomical structures? In: Biomedical Visualisation, Springer, 2019:1-11

4. Morrow DS, Cupp JA, Broder JS. Versatile, reusable, and inexpensive ultrasound phantom procedural trainers. J Ultrasound Med 2016;35:831-841.

5. Kwon SY, Hong SH, Kim ES, Park HJ, You Y, Kim YH. The efficacy of lumbosacral spine phantom to improve resident proficiency in performing ultrasound-guided spinal procedure. Pain Med 2015;16:2284-2291.

6. Scheppler JA. Watts cooking: using a microwave to prepare bacterial media for inquiry-based experiments. Staff Publications \& Research 2014;2. http://digitalcommons.imsa. edu/sir_staffpr/2.

\section{Sonography of Encapsulated Fat Necrosis with Histologic Correlation}

\section{Abdulhadi Jfri'1, Laura Najera ${ }^{2}$, Fernando Alfageme ${ }^{3}$}

${ }^{1}$ Division of Dermatology, McGill University Health Centre, Montreal General Hospital, Montreal, Canada, ${ }^{2}$ Pathology Department, Hospital Universitario Puerta de Hierro Majadahonda, Madrid, Spain, ${ }^{3}$ Dermatology Department, Hospital Universitario Puerta de Hierro Majadahonda, Madrid, Spain

\section{To the Editor,}

The pathogenesis of encapsulated fat necrosis is still not entirely clear, but the most accepted theory is that

Received 30.05.2020 Accepted 15.06.2020

Med Ultrason

2020, Vol. 22, No 3, 379-380, DOI: 10.11152/mu-2650,

Corresponding author: Fernando Alfageme, Assoc Prof Dermatology

Autonomous University of Madrid,

Calle Joaquín Rodrigo, 1 ,

28222 Majadahonda, Madrid, Spain

Phone: +34 911916000

E-mail: dermalfageme@gmail.com it is secondary to trauma and interruption of blood supply [1] that leads to a rapid infarction of adipose tissue lobules. In the following inflammatory response, necrotic adipose tissue separates from healthy tissue [2] as a result of macrophage involvement, and this leads to a capsule or fibrous pseudomembrane appearance, surrounding the coalesced necrotic, as a result of a fibrocellular response. The resulting lesions become mobile [2] when the capsule creates a cleavage plane between itself and the surrounding tissues. Histological analysis reveals that fibrous septa encapsulating the necrotic adipose tissue reflects calcification [3] and contains inflammatory cell 

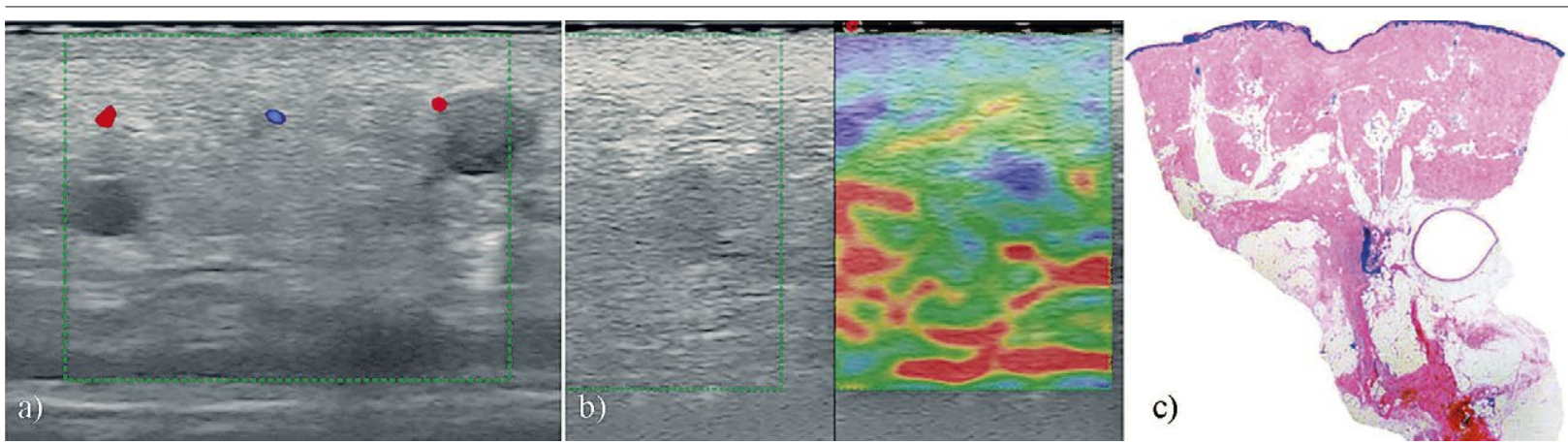

Fig 2. Multiple hypoechoic well limited spherical subcutaneous lesions ranging 4-5 mm in diameter with scarce perilesional vascularization (a). Shear-Wave Elastography demonstrated homogeneous hard lesional areas surrounded by soft subcutaneous tissue (b). Hematoxylin and eosin stain showing well circumscribed nodule, localized in the subcutaneous tissue surrounded by a thin hyalinized fibrous capsule (c).

infiltrates. The condition has also been associated with pancreatic disease. [4]

Encapsulated fat necrosis is often seen in fat-rich areas prone to trauma. The condition can present with a painful acute abdomen when located in the abdominal wall and either mimics appendicitis [5] or is found incidentally free in the intra-abdominal cavity [6]. The disease is curative with surgical excision and usually no risk of recurrence.

We present a 47-year-old female with known HIV infection being treated with high active antiretroviral therapy (HAART) that includes didanosine, nevirapine and ritonavir, with no new medication initiated recently. The patient presented with a few days' history of multiple painful lumpy lesions in the back. Lab tests showed normal amylase, lipase, lipid profile, calcium and undetectable viral load with CD4 count $800 \mathrm{~mm}^{3}$. Patient denied any history of trauma. Examination revealed multiple subcutaneous mobile tender nodules over the lumbar region. Ultrasound showed multiple hypoechoic well limited spherical subcutaneous lesions ranging in size from 4 to $5 \mathrm{~mm}$ maximum diameter, with scarce perilesional vascularization. Shear-Wave Elastography (SWE) demonstrated homogeneous hard lesional areas surrounded by soft subcutaneous tissue. Fine needle aspiration biopsy (FNAB) revealed fat. Wedge excisional biopsy was performed on one of the lesions and showed well circumscribed nodule, localized in the subcutaneous tissue surrounded by a thin hyalinized fibrous capsule with evidence of lobular panniculitis with fat necrosis (fig 1).
In diagnosing this condition, differential diagnoses include lipomas and liposarcoma. We believe that our case study is the first in which ultrasound is used to distinguish between encapsulated fat necrosis with histologic correlation and these other conditions. Because lipomas appear as a soft mass with variable echogenicity with wide inter-reader variability, mostly isoechoic followed by hyperechoic then hypoechoic [7] and liposarcomas can be multilobulated and present on ultrasound as multiple anechoic regions, we believe that ultrasound provides an effective method of diagnosing encapsulated fat necrosis.

\section{References}

1. Hurt MA, Santa Cruz DJ. Nodular-cystic fat necrosis. A reevaluation of the so-called mobile encapsulated lipoma. J Am Acad Dermatol 1989;21:493-498.

2. Diaz-Cascajo C, Borghi S. Subcutaneous pseudomembranous fat necrosis: new observations. J Cutan Pathol 2002;29:5-10.

3. Kiryu H, Rikihisa W, Furue M. Encapsulated fat necrosis - a clinicopathological study of 8 cases and a literature review. J Cutan Pathol 2000;27:19-23.

4. Szymanski FJ, Bluefarb SM. Nodular fat necrosis and pancreatic diseases. Arch Dermatol 1961;83:224229.

5. De Kock I, Delrue L. Encapsulated Mesenteric Fat Necrosis. J Belg Soc Radiol 2016;100:53.

6. Image of the Month-Diagnosis. Arch Surg 2011;146:1450.

7. Inampudi P, Jacobson JA, Fessell DP et al. Soft-tissue lipomas: accuracy of sonography in diagnosis with pathologic correlation. Radiology 2004;233:763-767. 


\title{
Use of ultrasound as an alternative diagnostic method for the detection of Meralgia Paresthetica
}

\author{
Mustafa Turgut Yildizgoren
}

Fizikon Medical Center, Department of Physical Medicine and Rehabilitation, Konya, Turkey

\section{To the Editor,}

Meralgia paresthetica (MP), also called lateral femoral cutaneous neuropathy, one of the most common mononeuropathies of the lower limb, is a sensory dysfunction often associated with injury, usually in the form of compression or entrapment of the lateral femoral cutaneous nerve (LFCN) [1]. Patients typically present with numbness, paresthesia and a burning sensation over the anterolateral aspect of the thigh, but there is no motor weakness or wasting. Common causes are obesity, pregnancy, wearing tight clothing, repetitive motion of the legs, or recent injuries of the hip. The compression/irritation is often due to the entrapment of the nerve by the inguinal ligament as it courses along the ASIS on its way to the thigh. Electrodiagnostic studies are often necessary for confirmation of the diagnosis of MP, but electrodiagnostic studies are generally not preferred for diagnosis in clinical practice. MP can usually be diagnosed clinically from the symptoms alone [2].

A 62-year old female presented at our Outpatients Clinic with complaints of loss of sensation and paresthesia in the left anterolateral thigh, which had been ongoing for three months. The pain worsened in the left upper thigh when she started walking. On examination, although the straight leg raising test and tendon reflexes were normal, hypoesthesia and paresthesia in the LFCN skin innervation area was detected. There was no motor weakness. Based on the medical history and physical examination, meralgia paresthetica was suspected, so an ultrasonographic evaluation of the LFCN was planned for

Received 21.05.2020 Accepted 09.06.2020

Med Ultrason

2020, Vol. 22, No 3, 381-382, DOI: 10.11152/mu-2549,

Corresponding author: Mustafa Turgut Yildizgoren, MD

Fizikon Medical Center, Department of

Physical Medicine and Rehabilitation

Konya, Turkey

Phone: 903323536900

Fax: 903323536900

E-mail: ftr.mustafaturgut@hotmail.com

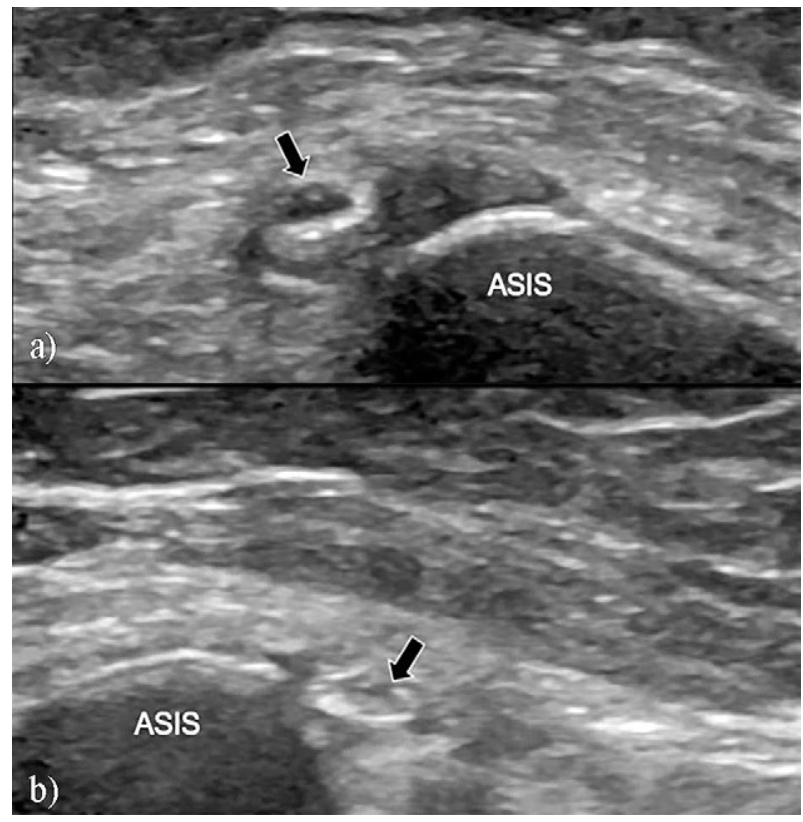

Fig 3. Transverse ultrasonographic image of the lateral femoral cutaneous nerve (LFCN) on the entrapment side (A), and the normal side (B). The LFCN seems to be swollen and hypoechoic where it passes medial to the anterior superior iliac spine (ASIS) and below the inguinal ligament.

the purpose of diagnosis and treatment. The linear probe (7-12MHz) was positioned transversely $1 \mathrm{~cm}$ inferior of the ASIS, and in line with the inguinal ligament it was moved down and the LFCN was identified as a swollen and hypoechoic structure (fig 1).

Recently, US has been increasingly used for the diagnosis of entrapment neuropathies and peripheral nerve injections. US has been shown to be particularly suitable for observation and injection to small and superficial structures [3]. US guidance allows for real-time direct visualization of a target nerve and nearby anatomic structures, the identification of possible anatomic variations, evaluation of the volume distribution of the local anesthetic, and prevents the risk of complications such as the inadvertent puncture of vessels. 


\section{References}

1. Boyd KU, Brown JM. Injury and compression neuropathy in the lower extremity. In: Mackinnon SE, Yee A. (Eds). Nerve surgery. New York, Thieme; 2015:338390 .
2. Parisi TJ, Mandrekar J, Dyck PJ, Klein CJ. Meralgia paresthetica: relation to obesity, advanced age, and diabetes mellitus. Neurology 2011;77:1538-1542.

3. Ozçakar L, Carli AB, Tok F, Tekin L, Akkaya N, Kara M. The utility of musculoskeletal ultrasound in rehabilitation settings. Am J Phys Med Rehabil 2013;92:805-817.

\title{
Ultrasound registry in Rheumatology: a first step into a foreseeable future
}

\author{
João Madruga Dias ${ }^{1,2}$, Annamaria Iagnocco ${ }^{3}$
}

${ }^{1}$ Rheumatology Department, Centro Hospitalar Médio Tejo, Torres Novas, Portugal, ${ }^{2}$ CEDOC, Nova Medical School, Lisbon, Portugal, ${ }^{3}$ Academic Rheumatology Centre, Università degli Studi di Torino, Turin, Italy

\section{To the Editor,}

Registries are paramount in Rheumatology, offering new opportunities for clinical and translational research. Many rheumatology registries are based on clinical inputs, also encompassing laboratory and imaging data (such as conventional radiography and MRI) [1]. The information present in registries is being targeted for measuring quality of care in rheumatology, allowing performance feedback and eventually tying rheumatologists' payments to measures of quality and cost [2].

Ultrasonography became essential in modern Rheumatology, with an explosion of research and clinical applications since the 2000's, changing the way we diagnose, follow-up and treat rheumatic diseases, and is progressively being applied to clinical trials $[3,4]$.

A search on PubMed for "ultrasound + registry + rheumatology" found 128 results with none being an existing dedicated rheumatology ultrasound registry. A search using "ultrasound + database + rheumatology" had the same result for 134 results, while "rheumatology + ultrasonography + registry" and "rheumatology + ultrasonography+ database" had 118 and 122 results,

Received 12.07.2020 Accepted 16.07.2020

Med Ultrason

2020, Vol. 22, No 3, 382-383, DOI: 10.11152/mu-2725,

Corresponding author: Dr. João Madruga Dias

Rheumatology Department,

Centro Hospitalar Médio Tejo,

Torres Novas, Portugal

E-mail: joao_alexandre@hotmail.com respectively, with no dedicated rheumatology ultrasound registry found.

In 2018 we developed a dedicated rheumatology ultrasound registry that would encompass all joints usually scanned by ultrasonography and also arteries and salivary glands. Being a multipurpose ultrasonography registry for Rheumatology application, it is useful for both clinical and research purposes. Since it is a prospective registry, it means patients' information is recorded at several time points, both on a clinical necessity basis and in defined settings such as prospective research projects and trials. Moreover, each patient is individually registered, allowing cross analysis with clinical, radiology and laboratory registries and further enhancing the retrieved information, exponentially increasing the applications for the ultrasound registry.

Since the beginning this registry was projected as an international initiative, with a first trial run in selected European countries. More than being a short-lived database for specific projects, it is meant to be a dynamic and encompassing registry for all ultrasound-related aspects in Rheumatology.

The human-input interface is as minimalistic and user-friendly as possible, with online access. Other than data input, storage, and participation in research projects, the registry is designed to deliver standardized ultrasonography reports based on the data input by the ultrasonographer, becoming a time-saving clinical tool and helping to systematize and standardize ultrasonography.

Each user has an individual login and can only upload and edit his/her own data (data retrieval from other 
centres is available on request for clinical or research purposes). Users have to present minimal ultrasonography training credentials (EULAR certificate for example) and/or pass an introductory assessment developed for interobserver agreeability. For specific projects or data inputs (elastography, paediatric rheumatology, for example) further requirements may be created giving access only to a more stringent group of users meeting specified requirements, enhancing data input quality.

The fact that can be used as a transversal tool across Rheumatology in Europe, promotes standardization of knowledge and procedures in ultrasonography, contributing to a more even learning progression at a transnational level. Overall, we think this will lead to better patient care in Rheumatology.

\section{References}

1. Elkayam O, Pavelka K. Biologic registries in rheumatology: lessons learned and expectations for the future. Autoimmun Rev 2012;12:329-336.

2. Tonner C, Schmajuk G, Yazdany J. A new era of quality measurement in rheumatology: electronic clinical quality measures and national registries. Curr Opin Rheumatol 2017;29:131-137.

3. Emery P, Horton S, Dumitru RB, et al. Pragmatic randomised controlled trial of very early etanercept and MTX versus MTX with delayed etanercept in RA: the VEDERA trial. Ann Rheum Dis 2020;79:464-471.

4. Lillegraven S, Haavardsholm EA. Subclinical Treatment Targets in Rheumatology: Lessons from Randomized Clinical Trials in Rheumatoid Arthritis. Rheum Dis Clin North Am 2019;45:593-604. 